\title{
Georessource Untergrund: Chancen und Risiken im Rahmen der Energiewende
}

\author{
Michael Kühn $^{1} \cdot$ Sonja Martens ${ }^{1}$
}

Online publiziert: 12. Mai 2016

(c) Springer-Verlag Berlin Heidelberg 2016

„Der Schutz des Grundwassers als Daseinsfürsorge ist wichtig und richtig. Die energetische Nutzung des Untergrunds, auch des Grundwassers und der Grundwasserleiter hat ebenfalls ihre Berechtigung und wird zukünftig immer wichtiger werden“ (Scheytt und Massmann 2016). Die Quintessenz des Editorials des vorherigen Heftes gilt unverändert auch für diese Ausgabe.

Wir möchten hier Beispiele für Chancen geben, die der geologische Untergrund für die Energiewende bietet, aber gleichzeitig auch damit verbundene Risiken aufzeigen. Ausgangsbasis ist das Energiekonzept der Bundesregierung für eine umweltschonende, zuverlässige und bezahlbare Energieversorgung. Darin festgelegt ist eine Reduktion der Treibhausgasemissionen bis zum Jahr 2050 um 80-95\%, bezogen auf den Ausstoß von 1990. Im gleichen Zeitraum soll der Anteil der regenerativen Energien am Bruttoenergieverbrauch in Deutschland auf $60 \%$ erhöht werden. Folgt man dem Modell des Fraunhofer-Instituts für Solare Energiesysteme (Henning und Palzer 2014), dann ist für Deutschland eine Energieversorgung mit Strom und Wärme zu $100 \%$ über regenerative Energie möglich. In den quantitativen Modellen werden ausschließlich bereits verfügbare Technologien innerhalb ihrer bekannten Potenzialgrenzen betrachtet. Aufgrund der Nichtplanbarkeit der Energieerzeugung aus Sonne und Wind werden Speicher wie Pumpspeicher-Kraftwerke, Batterien, Wärme- und Methangasspeicher berücksichtigt. Zudem ist oberflächennahe Geothermie über elektrische Wärmepumpen in das System integriert.

Michael Kühn

michael.kuehn@gfz-potsdam.de

1 Deutsches GeoForschungsZentrum GFZ, Potsdam, Deutschland
Das theoretische geothermische Potenzial Deutschlands errechnet sich aus der Wärmemenge, die in prinzipiell erbohrbarer Tiefe gespeichert ist. Diese Wärmemenge entspricht etwa 3-mal dem aktuellen weltweiten und 115-mal dem deutschen Verbrauch. Dieses theoretische Potenzial ist jedoch aufgrund einer Vielzahl von Restriktionen nicht erschließbar. Beispielsweise sind Flächen, die von potenziellen Verbrauchern weit entfernt liegen, wegen zu hoher Verluste beim Energietransport für eine Erdwärmenutzung ungeeignet. Nachhaltig für Deutschland wäre Geothermie, wenn nur der permanente natürliche Wärmestrom, der sich oberflächennah durch Sonneneinstrahlung sowie dem Wärmestrom aus dem Erdinneren ergibt, genutzt würde. Diese Leistung lässt sich erhöhen, wenn neben der Wärmenutzung im Winter auch im Sommer durch Gebäudekühlung im Überschuss vorhandene Wärme in den Untergrund zurückgespeichert wird.

Untergrundgasspeicherung ist Stand der Technik. Die aktuelle Kapazität aller Untergrundgasspeicher in Deutschland liegt bei 24 Milliarden Norm-Kubikmetern Methangas. Diese Menge entspricht 3-mal jener im Modell des Fraunhofer-Instituts zugrunde gelegten zu speichernden Energie. Untertagegasspeicher sind zudem eine Option als bevorratendes Bindeglied bei der Speicherung von Strom aus regenerativen Energien. Wesentliche Grundlage dabei ist das „Power to Gas“-Verfahren. Überschussenergie lässt sich über Wasserstoff zusammen mit Kohlenstoffdioxid in Methan (synthetisches Erdgas) umwandeln, welches man bei Bedarf nutzen kann. Für Deutschland ergibt sich eine Kapazität für die Technologie basierend auf der aktuellen Anzahl bereits vorhandener Gasspeicher von 20-60\% der 90-270 TWh Überschussenergie, die für das Jahr 2050 erwartet werden (Kühn et al. 2013).

Ein Risiko, das man bei der Nutzung des unterirdischen Raums eingeht, ist die induzierte Seismizität. Die stärks- 
ten Beben, die in Deutschland bislang aufgezeichnet wurden, sind natürliche, geologische Ereignisse im Bereich MW 6-7. Der Mensch spürt Magnituden kleiner 3 generell nicht. Bei der Erdgasförderung, beim Kohle- und Erzbergbau sowie durch den Betrieb von Staudämmen lagen die Messungen maximal im Bereich MW 4-5, beim Salzbergbau bei maximal MW 5-6. Im Rahmen geothermischer Erkundung oder Nutzung des Untergrundes lagen die Beben bislang immer unterhalb von MW 4 (Grünthal 2014).

Bohrungen sind potenzielle Leckagewege, weil durch sie Wegsamkeiten im Untergrund hergestellt werden. Es gibt industrielle Erfahrungen aus mehr als 4 Millionen Ölund Gas-Bohrungen rund um die Welt. Einerseits kann jede Bohrung theoretisch versagen und Leckagen verursachen, andererseits aber durch Reparaturmaßnahmen auch wiederhergestellt werden. Von den 4 Millionen Bohrungen sind 25 Dokumentationen von insgesamt 400.000 zugänglich. Darin wird je nach Nutzung in 1,9-75\% der Fälle ein Versagen der Zementierung oder der Verrohrung berichtet (Davies et al. 2014).

Der geologische Untergrund liefert in signifikanter Größenordnung einerseits Trinkwasser und die klassischen Rohstoffe Erdöl und Erdgas. Andererseits steht Speicherplatz für Gas, Wärme und Kälte zur Verfügung (Martens und Kühn 2015). Unsere Aufgabe als Geowissenschaft- ler und Ingenieure ist es, die Antwort auf die Frage zu geben, wie die Georessourcen und der Untergrund verantwortungsvoll und nachhaltig genutzt und zur Realisierung der Ziele im Rahmen der Umsetzung der Energiewende herangezogen werden können.

\section{Literatur}

Davies, R.J., Almond, S., Ward, R.S., Jackson, R.B., Adams, C., Worrall, F., Herringshaw, L.G., Gluyas, J.G., Whitehead, M.A.: Oil and gas wells and their integrity: Implications for shale and unconventional resource exploitation. Mar Pet Geol 56, 239-254 (2014)

Grünthal, G.: Induced seismicity related to geothermal projects versus natural tectonic earthquakes and other types of induced seismic events in Central Europe. Geothermics 52, 22-35 (2014)

Henning, H.M., Palzer, A.: A comprehensive model for the German electricity and heat sector in a future energy system with a dominant contribution from renewable energy technologies - Part I: Methodology. Renew Sustain Energy Rev 30, 1003-1018 (2014)

Kühn, M., Nakaten, N., Streibel, M., Kempka, T.: Klimaneutrale Flexibilisierung regenerativer Überschussenergie mit Untergrundspeichern. Erdöl Erdgas Kohle 129(10), 348-352 (2013)

Martens, S., Kühn, M.: Geological underground will contribute significantly to the implementation of the energy policy towards Renewables in Germany. Energy Procedia 76, 59-66 (2015)

Scheytt, T., Massmann, G.: Im Spannungsfeld von Wasserwirtschaft und Energie. Grundwasser 21(1), 1 (2016) 